\title{
HYPERFINITE GRAPH LIMITS
}

\author{
ODED SCHRAMM
}

(Communicated by Krzysztof Burdzy)

\begin{abstract}
Gábor Elek introduced the notion of a hyperfinite graph family: a collection of graphs is hypefinite if for every $\epsilon>0$ there is some finite $k$ such that each graph $G$ in the collection can be broken into connected components of size at most $k$ by removing a set of edges, of size at most $\epsilon|V(G)|$. We presently extend this notion to a certain compactification of finite boundeddegree graphs and show that if a sequence of finite graphs converges to a hyperfinite limit, then the sequence itself is hyperfinite.
\end{abstract}

\section{INTRODUCTION}

While studying asymptotic properties of finite graphs, it can be worthwhile to introduce compactifications of various collections of finite graphs. In one such compactification, which is particularly well suited to bounded degree graphs, the notion of convergence is local weak convergence, as studied in [6] and [2]. A different notion of convergence [12] is suitable for dense graphs, in which the number of edges is roughly proportional to $|V(G)|^{2}$. Here, we will focus on the bounded degree case.

The purpose of this note is to extend the study of the local weak convergence of graphs, whose definition will be recalled below, by noting its properties in relation to the collection of hyperfinite graphs. A collection of finite graphs $\mathcal{G}$ is $(k, \epsilon)$ hyperfinite if every $G \in \mathcal{G}$ has a set of edges $S \subset E(G)$ such that $|S| \leq \epsilon|V(G)|$ and every connected component of $G \backslash S$ has at most $k$ vertices. We say that $\mathcal{G}$ is hyperfinite, if for every $\epsilon>0$ there is some finite $k=k(\epsilon)$ such that $\mathcal{G}$ is $(k, \epsilon)$ hyperfinite. Many interesting collections of graphs are hyperfinite. For example, as noted in [13, Theorem 3], it follows from the planar separator theorem that the set of planar graphs with maximal degree at most $M$ is hyperfinite for every $M<\infty$.

Although the notion of hyperfiniteness appears implicitly in the literature (for example, [13]), as far as we know Gábor Elek [8, 9, 10] was the first to give it a name and propose its systematic study.

We now prepare to introduce the notion of local weak convergence. A rooted graph is a pair $(G, o)$, where $o \in V(G)$. An isomorphism of rooted graphs $\phi:(G, o) \rightarrow\left(G^{\prime}, o^{\prime}\right)$ is an isomorphism of the underlying graphs which satisfies $\phi(o)=o^{\prime}$. A graph is locally-finite if each vertex is incident to only finitely many

Received by the editors December 27, 2007.

2000 Mathematics Subject Classification. Primary 60C05; Secondary 05 C 80.

Key words and phrases. Graph limits, generalizations of amenability.

We are grateful to Gábor Elek, Christian Borgs and Russell Lyons for beneficial conversations.

(C)2008 American Institute of Mathematical Sciences 17

I. Benjamini, O. Häggström (eds.), Selected Works of Oded Schramm, Selected Works in Probability and Statistics, DOI 10.1007/978-1-4419-9675-6_19, C Springer Science+Business Media, LLC 2011 
edges. Let $\widehat{\mathcal{G}}$ denote the collection of all isomorphism classes of connected, locallyfinite rooted graphs. For $M \in \mathbb{N}_{+}$, let $\widehat{\mathcal{G}}_{M} \subset \widehat{\mathcal{G}}$ denote the subcollection consisting of rooted graphs with maximal degree at most $M$. For $(G, o) \in \widehat{\mathcal{G}}$ and $r \geq 0$, let $B_{G}(o, r)$ denote the subgraph of $G$ spanned by the vertices at distance at most $r$ from $o$. If $(G, o),\left(G^{\prime}, o^{\prime}\right) \in \widehat{\mathcal{G}}$ and $r$ is the largest integer such that $\left(B_{G}(o, r), o\right)$ is rooted-graph isomorphic to $\left(B_{G^{\prime}}\left(o^{\prime}, r\right), o^{\prime}\right)$, then we set $\rho\left((G, o),\left(G^{\prime}, o^{\prime}\right)\right)=1 / r$. We also set $\rho((G, o),(G, o))=0$. Then $\rho$, thus defined, is a metric on $\widehat{\mathcal{G}}$. Let $\mathfrak{M}_{M}$ (respectively $\mathfrak{M}$ ) denote the space of all probability measures on $\widehat{\mathcal{G}}_{M}$ (resp. $\widehat{\mathcal{G}}$ ) that are measurable with respect to the Borel $\sigma$-field of $\rho$. Then $\mathfrak{M}_{M}$, endowed with the topology of weak convergence, is compact.

Let $\mathcal{G}_{M}^{0}$ denote the collection of all isomorphism classes of finite graphs with maximal degree at most $M$ and let $G \in \mathcal{G}_{M}^{0}$. Let $o \in V(G)$ be chosen randomlyuniformly and let $G_{o}$ be the connected component of $o$. Then the law (of the isomorphism type) of $\left(G_{o}, o\right)$ is an element of $\mathfrak{M}_{M}$, which will be denoted by $\Psi(G)$. This defines a mapping, $\Psi: \mathcal{G}_{M}^{0} \rightarrow \mathfrak{M}_{M}$, whose restriction to the set of connected graphs is injective.

Let $\mathfrak{M}_{M}^{0}$ denote the closure of $\Psi\left(\mathcal{G}_{M}^{0}\right)$ in $\mathfrak{M}_{M}$. It has been observed in [6] that every $\mu \in \mathfrak{M}_{M}^{0}$ satisfies an intrinsic version of the Mass Transport Principle (MTP). The MTP was invented by Olle Häggström in the setting of regular trees [11], and has been used extensively by Benjamini, Lyons, Peres and Schramm in the more general setting of transitive and quasi-transitive graphs [4]. Since then, there have been numerous successful applications of the MTP. A measure, $\mu \in \mathfrak{M}$, satisfies the intrinsic MTP (iMTP) if for every non-negative function $f(G, x, y)$, which takes as arguments a connected graph and two vertices in it, and depends only on the isomorphism type of the triple $(G, x, y)$, we have

$$
\int \sum_{x \in V(G)} f(G, x, o) d \mu(G, o)=\int \sum_{y \in V(G)} f(G, o, y) d \mu(G, o) .
$$

Let $\mathcal{U}$ denote the set of $\mu \in \mathfrak{M}$ satisfying the iMTP and set $\mathcal{U}_{M}:=\mathcal{U} \cap \mathfrak{M}_{M}$. The easy proof that $\mathfrak{M}_{M}^{0} \subset \mathcal{U}$ proceeds by first noting that $\Psi\left(\mathcal{G}_{M}^{0}\right) \subset \mathcal{U}$ and then observing that $\mathcal{U}_{M}$ is closed in $\mathfrak{M}_{M}$.

Aldous and Lyons [2] coined the term unimodular for measures in $\mathcal{U}$, and studied their properties. They also raised the fundametal problem of determining whether $\mathfrak{M}_{M}^{0}=\mathcal{U}_{M}$; that is, whether every $\mu \in \mathcal{U}_{M}$ can be approximated by elements of $\Psi\left(\mathcal{G}_{M}^{0}\right)$.

If $\nu$ is a measure on triples $(G, o, S)$, where $G$ is a connected graph, $o \in V(G)$, and $S$ is some structure on $G$ (such as a labeling of the edges or vertices, or a subgraph, etc), then $\nu$ is unimodular if it satisfies the above iMTP, where $f$ is allowed to depend on $S$.

A measure $\mu \in \mathcal{U}$ is $(k, \epsilon)$-hyperfinite if there is a measure, $\nu$, on triples, $(G, o, S)$, such that the projection of $\nu$ to $(G, o)$ is $\mu, S \subset E(G)$, every connected component of $G \backslash S$ has at most $k$ vertices, $\nu$ is unimodular and the $\nu$-expected number of edges in $S$ adjacent to $o$ is at most $2 \epsilon$.

The reason for choosing $2 \epsilon$ instead of $\epsilon$ is the following. If $G_{0}$ is a finite graph which is $(k, \epsilon)$-hyperfinite and $\mu=\Psi\left(G_{0}\right)$, then $\mu$ is $(k, \epsilon)$-hyperfinite. This is because if $S_{0} \subset E\left(G_{0}\right)$ satsifies $\left|S_{0}\right| \leq \epsilon\left|V\left(G_{0}\right)\right|$, then the uniform law on the triples $\left(G_{0}, o, S_{0}\right)$, where $o \in V\left(G_{0}\right)$, is unimodular and the expected number of edges of 
$S_{0}$ adjacent to $o$ is $2\left|S_{0}\right| /|V(G)| \leq 2 \epsilon$. Conversely, we show in Lemma 2.1 below that if $G \in \mathcal{G}_{M}^{0}$ and $\Psi(G)$ is $(k, \epsilon)$-hyperfinite, then $G$ is also $(k, \epsilon)$-hyperfinite.

A measure $\mu \in \mathcal{U}$ is hyperfinite if, for every $\epsilon>0$, there is a finite $k=k(\epsilon)$ such that $\mu$ is $(k, \epsilon)$-hyperfinite.

In this paper we will prove the following theorem.

Theorem 1.1. Let $M \in \mathbb{N}_{+}$, and let $G_{1}, G_{2}, \ldots$ be a sequence in $\mathcal{G}_{M}^{0}$ with $\Psi\left(G_{j}\right)$ converging weakly to some $\mu \in \mathfrak{M}_{M}$. Then $\mu$ is hyperfinite if and only if the sequence $\left\{G_{1}, G_{2}, \ldots\right\}$ is hyperfinite.

In the forthcoming paper [7] we give an application of this theorem to planarity testing.

The easy direction of the theorem is to show that $\mu$ is hyperfinite if $\left\{G_{1}, G_{2}, \ldots\right\}$ is hyperfinite. The opposite direction is an immediate consequence of the following, more quantitive, version.

Theorem 1.2. Suppose that $\mu \in \mathcal{U}_{M}$ is $(k, \epsilon)$-hyperfinite, where $0<\epsilon<1$ and $k<\infty$. Then there is some open neighborhood of $\mu$ such that every $\mu^{\prime} \in \mathcal{U}_{M}$ within that neighborhood is $(k, \tilde{\epsilon})$-hyperfinite, where

$$
\tilde{\epsilon}:=3 \log (2 M / \epsilon) \epsilon .
$$

Theorem 1.2 is quantitative in the sense that the dependence of $\tilde{\epsilon}$ on $\epsilon$ is explicit. In [7], we present a finitary variant of Theorem 1.2 which bounds the size of the neighborhood. The proof there is also a finitary variant of the proof below.

I. Benjamini (private communication) points out that hyperfiniteness of measures in $\mathcal{U}_{M}$ is an appropriate analogue for amenability in the setting of transitive graphs. In particular, the Burton-Keane [3] argument can show that percolation on a sample from $\mathcal{U}_{M}$ can produce at most one infinite cluster a.s. Extending a conjecture from [5] in the transitive setting, Benjamini also conjectures the converse: that if $\mu \in \mathcal{U}_{M}$ is not hyperfinite, then there is some parameter $p \in[0,1]$ such that $\operatorname{Bernoulli}(p)$ percolation on the sample from $\mu$ has more than one infinite cluster, with positive probability. See [1] for a related discussion in the context of percolation on finite graphs and, in particular, the discrete hypercube.

\section{Proofs}

As a warm up, we prove the following.

Lemma 2.1. Let $\epsilon>0, k \in \mathbb{N}_{+}$and $G \in \mathcal{G}_{M}^{0}$. Then $G$ is $(k, \epsilon)$-hyperfinite if and only if $\Psi(G)$ is $(k, \epsilon)$-hyperfinite.

Proof. The "only if" direction was sketched in the introduction, and hence we presently only prove the "if" direction. Assume that $\Psi(G)$ is $(k, \epsilon)$-hyperfinite. Then there is a unimodular probability measure $\nu$ on triples $\left(G^{\prime}, S, o\right)$ such that $G^{\prime}$ is $\nu$-a.s. a connected component of $G, S \subset E\left(G^{\prime}\right), o \in V\left(G^{\prime}\right)$, the $\nu$-distribution of $o$ is uniform in $V(G)$, the connected components of $G^{\prime} \backslash S$ all have at most $k$ vertices $\nu$-a.s. and the $\nu$-expected number of edges in $S$ that are adjacent to $o$ is at most $2 \epsilon$.

Let $S(v)$ denote the edges of $S$ incident to a vertex $v$. Let $G_{1}, G_{2}, \ldots, G_{m}$ be the connected components of $G$. Let $\mathcal{A}_{i}$ be the event that $G^{\prime}$ is isomorphic to $G_{i}$. Define $g\left(G^{\prime}, S, x, y\right)$ to be the number of edges of $S$ that are incident to $x$ and 
$f_{i}\left(G^{\prime}, S, x, y\right)=1_{\mathcal{A}_{i}} g\left(G^{\prime}, S, x, y\right)$. Since $\nu$ is unimodular, the iMTP may be applied to $f_{i}$ :

$$
\int \sum_{y \in V\left(G^{\prime}\right)} f_{i}\left(G^{\prime}, S, o, y\right) d \nu=\int \sum_{x \in V\left(G^{\prime}\right)} f_{i}\left(G^{\prime}, S, x, o\right) d \nu .
$$

The left hand side of (2.1) is equal to

$$
\nu\left[|S(o)|\left|V\left(G^{\prime}\right)\right| 1_{\mathcal{A}_{i}}\right]=\left|V\left(G_{i}\right)\right| \nu\left[|S(o)| 1_{\mathcal{A}_{i}}\right],
$$

while the right hand side is $\nu\left[2|S| 1_{\mathcal{A}_{i}}\right]$. Hence,

$$
\left|V\left(G_{i}\right)\right| \nu\left[|S(o)| 1_{\mathcal{A}_{i}}\right]=\nu\left[2|S| 1_{\mathcal{A}_{i}}\right] .
$$

Therefore, there is some $S_{i} \subset E\left(G_{i}\right)$ such that each connected component of $G_{i} \backslash S_{i}$ has at most $k$ vertices and

$$
\left|S_{i}\right| \nu\left[\mathcal{A}_{i}\right] \leq \frac{\left|V\left(G_{i}\right)\right|}{2} \nu\left[|S(o)| 1_{\mathcal{A}_{i}}\right] .
$$

Define $\tilde{S}:=\bigcup_{i=1}^{m} S_{i}$. Note that $\nu\left[\mathcal{A}_{i}\right]|V(G)|$ is the number of vertices of $G$ that are in connected components of $G$ that are isomorphic to $G_{i}$. Therefore $t_{i}:=\nu\left[\mathcal{A}_{i}\right]|V(G)| /\left|V\left(G_{i}\right)\right|$ is the number of connected components of $G$ that are isomorphic to $G_{i}$. Note that

$$
\sum_{i=1}^{m} \frac{1_{\mathcal{A}_{i}}}{t_{i}}=1
$$

Dividing (2.2) by $\nu\left[\mathcal{A}_{i}\right]$ and summing over $i$ gives

$$
|\tilde{S}| \leq \frac{|V(G)|}{2} \nu\left[|S(o)| \sum_{i} \frac{1_{\mathcal{A}_{i}}}{t_{i}}\right]=\frac{|V(G)|}{2} \nu[|S(o)|] \leq 2 \epsilon|V(G)|,
$$

which completes the proof.

The idea of the proof of Theorem 1.2 is to replace the random set of edges, $S$, by a set of edges that still separates $G$ into connected components of size at most $k$, but has the extra feature that, for any $e \in E(G)$, the event $e \in S$ depends only on the local structure of $G$ near $e$ and some randomness. Thus, it is easy to adapt the law of this new $S$ to every random $G^{\prime}$ sufficiently close to $G$.

The proof of the theorem is complicated by a completely uninteresting point of a technical nature. For the sake of simplicity, we choose to present a proof that is not entirely precise, but does convey the essential ideas, and can be adapted to be completely correct. (See, [7].)

Proof of Theorem 1.2. Fix some $\epsilon_{0} \in(0,1 / 2)$. Let $\mathbf{P}$ be a unimodular measure on triples $(G, S, o)$ such that the marginal law of $(G, o)$ is $\mu$, the $\mathbf{P}$-expected number of edges of $S$ incident to $o$ is at most $2 \epsilon$, and every connected component of $G \backslash S$ has at most $k$ vertices.

For any $K \subset V(G)$ and any $v \in K$, let $p(K):=\mathbf{P}[K(v)=K \mid(G, o)]$. (Here, we are not entirely precise, since the expression $\mathbf{P}[K(v)=K \mid(G, o)]$ does not have the standard meaning of the probability of an event conditional on a $\sigma$-field. This is because $K$ and $v$ do not, a priori, have a meaning without $G$. This difficulty is not too hard to overcome, at the expense of obfuscating the proof.) Clearly, $p(K)$ does not depend on the choice of the particular $v \in K$. Moreover, a simple argument using the iMTP shows that $p(K)$ is not effected by a change of the basepoint $o$; in other words, $p(K)$ is really a function of the isomorphism type of the pair $(K, G)$. 
Given $(G, o)$ and $v \in V(G)$, let $\mathfrak{K}_{v}$ denote the collection of all connected sets of vertices, of cardinality at most $k$, that contain $v$. Obviously, $\left|\mathfrak{K}_{v}\right|$ is bounded by a function of $k$ and $M$. For all $r \in \mathbb{N}_{+}$, let $\mathcal{B}_{r}$ denote the (isomorphism type of the) rooted graph $(B(o, r), o)$. For $r>k$ and $K \in \mathfrak{K}_{o}$, we set

$$
p_{r}(K):=\mathbf{P}\left[K(o)=K \mid \mathcal{B}_{r}\right] .
$$

Clearly, $p_{r}(K)$ is a bounded martingale with respect to $r$ and $\lim _{r \rightarrow \infty} p_{r}(K)=p(K)$ a.s. Consequently, there is some finite $R$ such that

$$
\mathbf{E} \sum_{K \in \mathfrak{K}_{o}}\left|p_{R}(K)-p(K)\right|<\epsilon_{0} .
$$

Fix such an $R$.

For $K \in \mathfrak{K}_{o}$ let

$$
N_{R}(K):=\bigcup_{v \in K} B(v, R), \quad \tilde{p}(K)=\tilde{p}(K, G):=\mathbf{P}\left[K=K(o) \mid N_{R}(K)\right] .
$$

By unimodularity, $\tilde{p}(K)$ does not depend on the choice of basepoint $o$ in $K$. Therefore, by changing the basepoint, this also defines $\tilde{p}(K)$ for all $\mathfrak{K}_{v}, v \in V(G)$. Note that $\tilde{p}$ is a function whose arguments are a graph and a connected set of size at most $k$ in it and that $\tilde{p}$ depends only on the $R$-neighborhood of the set. However, $\tilde{p}$ is only defined if its arguments can occur in $(G, o)$. We extend the definition of $\tilde{p}$ to such arguments that have probability 0 of occuring in $(G, o)$ (if such exist) by setting it equal to 1 on such arguments.

Let $\mathfrak{K}:=\bigcup_{v \in V(G)} \mathfrak{K}_{v}$. Given $(G, o)$, let $\left(X_{K}: K \in \mathfrak{K}\right)$ be independent random variables such that

$$
\mathbf{P}\left[X_{K}=1 \mid(G, o)\right]=\min \left\{2 \log \left(1 / \epsilon_{0}\right) \tilde{p}(K), 1\right\}
$$

and $X_{K} \in\{0,1\}$ a.s. Let $F:=\bigcup_{K: X_{K}=1} \partial K$, where $\partial K$ denotes the edge-boundary of $K$, and let $W:=\bigcup\left\{K \in \mathfrak{K}: X_{K}=1\right\}$. Let $\tilde{F}$ denote the set of all edges incident to some vertex in $V(G) \backslash W$ and set $S^{\prime}:=F \cup \tilde{F}$. It is easy to verify that the law of $\left(G, S^{\prime}, o\right)$ is unimodular. Also, it is obvious that every connected component of $G \backslash S^{\prime}$ has cardinality at most $k$.

Our present goal is to estimate $\mathbf{E}\left[\left|E(o) \cap S^{\prime}\right|\right]$. We start by estimating $\mathbf{E}[|E(o) \cap \tilde{F}|]$. Let $\mathcal{A}$ denote the event $\sum_{K \in \mathfrak{R}_{o}} \tilde{p}(K)<1 / 2$.

\section{Lemma 2.2.}

$$
\mathbf{P}[\mathcal{A}]<2 \epsilon_{0} .
$$

Proof. Fix some $K \in \mathfrak{K}_{o}$. Since the sequence $p_{R}(K), \tilde{p}(K), p(K)$ is a martingale given $B(o, k)$, we have

$$
\mathbf{E}[|\tilde{p}(K)-p(K)| \mid B(o, k)] \leq \mathbf{E}\left[\left|p_{R}(K)-p(K)\right| \mid B(o, k)\right] .
$$

Our choice of $R$ therefore gives

$$
\mathbf{E}\left[\sum_{K \in \mathfrak{K}_{o}}|\tilde{p}(K)-p(K)|\right]<\epsilon_{0} .
$$

Since $\sum_{K \in \mathfrak{K}_{o}} p(K)=1$, we get

$$
\mathbf{E}\left[\left|1-\sum_{K \in \mathfrak{K}_{o}} \tilde{p}(K)\right|\right]<\epsilon_{0}
$$

and Markov's inequality completes the proof of the lemma. 


\section{Lemma 2.3.}

$$
\mathbf{P}[o \notin W]<3 \epsilon_{0} .
$$

Proof. Given $(G, o)$, the random variables $X_{K}$ are independent and satisfy $\mathbf{P}\left[X_{K}=1 \mid(G, o)\right]=\min \left\{1,2 \log \left(1 / \epsilon_{0}\right) \tilde{p}(K)\right\}$. Hence,

$$
\begin{aligned}
\mathbf{P}[o \notin W \mid(G, o)] & =\prod_{K \in \mathfrak{K}_{o}}\left(1-\mathbf{P}\left[X_{K}=1 \mid(G, o)\right]\right) \\
& \leq \exp \left(-\sum_{K \in \mathfrak{R}_{o}} 2 \log \left(1 / \epsilon_{0}\right) \tilde{p}(K)\right) \\
& \leq \exp \left(-2 \log \left(1 / \epsilon_{0}\right)(1 / 2)\right)+1_{\mathcal{A}}=\epsilon_{0}+1_{\mathcal{A}} .
\end{aligned}
$$

Now Lemma 2.2 completes the proof.

The lemma implies

$$
\mathbf{E}[|E(o) \cap \tilde{F}|] \leq 3 M \epsilon_{0},
$$

where $M$ is the bound on the degrees in $G$.

Now,

$$
\begin{aligned}
\mathbf{E}[|E(o) \cap F| \mid(G, o)] & \leq \sum_{K \in \mathfrak{K}_{o}}|\partial K \cap E(o)| \mathbf{P}\left[X_{K}=1 \mid(G, o)\right] \\
& \leq 2 \log \left(1 / \epsilon_{0}\right) \sum_{K \in \mathfrak{K}_{o}}|\partial K \cap E(o)| \tilde{p}(K) .
\end{aligned}
$$

Since,

$$
\mathbf{E}\left[\sum_{K \in \Re_{o}}|\partial K \cap E(o)| \tilde{p}(K)\right]=\mathbf{E}[|E(o) \cap \partial K(o)|]=\mathbf{E}[E(o) \cap S] \leq 2 \epsilon,
$$

we get

$$
\mathbf{E}[|E(o) \cap F|] \leq 4 \log \left(1 / \epsilon_{0}\right) \epsilon .
$$

Thus, we have,

$$
\mathbf{E}\left[\left|E(o) \cap S^{\prime}\right|\right] \leq 4 \log \left(1 / \epsilon_{0}\right) \epsilon+3 \epsilon_{0} M .
$$

We now choose $\epsilon_{0}:=\epsilon /(2 M)$.

Observe that the set $S^{\prime}$ is chosen in a very local way. Namely, given $(G, o)$, you can decide if $e \in S^{\prime}$ based purely on a fixed radius neighborhood of $e$ and some coin flips that are associated with this neighborhood. Given another unimodular $(\tilde{G}, \tilde{o})$, we can choose a $\tilde{S} \subset E(\tilde{G})$ according to the same procedure as $S^{\prime}$ is obtained for $G$. If $(\tilde{G}, \tilde{o})$ is sufficiently close to $(G, o)$, then the expected size of the set of edges in $\tilde{S}$ adjacent to $\tilde{o}$ will be close to the corresponding quantity in $G$. This proves the theorem.

Proof of Theorem 1.1. It is easy to verify that the set of $\nu \in \mathcal{U}_{M}$ that are $(k, \epsilon)$ hyperfinite is closed. Therefore, Lemma 2.1 implies that $\mu$ is hyperfinite if $\left\{G_{1}, G_{2}, \ldots\right\}$ is hyperfinite. The converse follows immediately from Theorem 1.2 and Lemma 2.1 . 


\section{REFERENCES}

[1] O. Angel and I. Benjamini, A Phase Transition for the Metric Distortion of Percolation on the Hypercube, 2003, arXiv:math/0306355

[2] D. Aldous and R. Lyons, Processes on Unimodular Random Networks, 2006, arXiv:math/0603062

[3] R. M. Burton and M. Keane. Density and uniqueness in percolation, Comm. Math. Phys., 121 (1989), 501-505. MR 0990777 (90g:60090)

[4] I. Benjamini, R. Lyons, Y. Peres and O. Schramm. Group-invariant percolation on graphs, Geom. Funct. Anal., 9 (1999), 29-66. MR 1675890 (99m:60149)

[5] Itai Benjamini and Oded Schramm. Percolation beyond $\mathbf{Z}^{d}$, many questions and a few answers, Electron. Comm. Probab., 1 (1996), 71-82 (electronic). MR 1423907 (97j:60179)

[6] Itai Benjamini and Oded Schramm. Recurrence of distributional limits of finite planar graphs, Electron. J. Probab., 6 (2001) (electronic). MR 1873300 (2002m:82025)

[7] I. Benjamini, O. Schramm and A. Shapira. Every minor-closed property of sparse graphs is testable, 2007. Preprint.

[8] G. Elek, The combinatorial cost, 2006, arXiv:math/0608474

[9] G. Elek, $L^{2}$-spectral invariants and convergent sequences of finite graphs, 2007, arXiv:0709.1261

[10] G. Elek, A regularity lemma for bounded degree graphs and its applications: Parameter testing and infinite volume limits, 2007, arXiv:0711.2800.

[11] Olle Häggström, Infinite clusters in dependent automorphism invariant percolation on trees, Ann. Probab., 25 (1997), 1423-1436. MR 1457624 (98f:60207)

[12] László Lovász and Balázs Szegedy, Limits of dense graph sequences, J. Combin. Theory Ser. B, 96 (2006), 933-957. MR 2274085 (2007m:05132)

[13] Richard J. Lipton and Robert Endre Tarjan, Applications of a planar separator theorem, SIAM J. Comput., 9 (1980), 615-627. MR 0584516 (82e:68067)

Microsoft Corporation, One Microsoft Way, Redmond, WA 98074, USA

E-mail address: schramm@microsoft.com 